\title{
Extreme Relations for Topological Flows
}

\author{
by
}

\author{
Brunon KAMIŃSKI, Artur SIEMASZKO and Jerzy SZYMAŃSKI
}

\author{
Presented by Andrzej LASOTA
}

\begin{abstract}
Summary. We introduce the concept of an extreme relation for a topological flow as an analogue of the extreme measurable partition for a measure-preserving transformation considered by Rokhlin and Sinai, and we show that every topological flow has such a relation for any invariant measure. From this result, it follows, among other things, that any deterministic flow has zero topological entropy and any flow which is a $K$-system with respect to an invariant measure with full support is a topological $K$-flow.
\end{abstract}

1. Introduction. The theory of invariant partitions discovered by Rokhlin and Sinai (cf. [13], [14]) is an important part of ergodic theory. Extreme and perfect partitions have found applications in ergodic theory and statistical mechanics. On the other hand, topological analogues of some concepts from this theory, for example, topological entropy and Pinsker relations, are useful tools in topological dynamics.

In our previous paper (cf. [9]), we defined the concept of a deterministic topological flow and of a topological $K$-flow using invariant closed equivalence relations as natural analogues of invariant measurable partitions. We also proved some basic properties of them.

In this note, we introduce the concept of an extreme relation which is an analogue of the extreme measurable partition of a Lebesgue space. From our main result, it follows (Corollary 1 ) that for any invariant measure $\mu$ there exists an extreme relation with respect to $\mu$. Hence, for a uniquely ergodic flow there exists an extreme relation. This result may by considered as a topological analogue of the Rokhlin-Sinai theorem (cf. [14]).

2000 Mathematics Subject Classification: Primary 37B05; Secondary 37B40, 37A35.

Key words and phrases: extreme relations, Pinsker relation, topological entropy, entropy pairs, deterministic flows, $K$-flows.

Research of B. Kamiński and J. Szymański supported by KBN grant 1 P03A 03826.

Research of A. Siemaszko partially supported by the KBN grant 1 P03A 03826. 
It follows that uniquely ergodic topological flows which have uniformly positive entropy are topological $K$-flows.

As another corollary of our Theorem, we obtain a new complete proof of Theorem 4 of [9] which says that any deterministic flow has zero entropy. Unfortunately, the original proof given in [9] has a gap.

We also show that if a topological flow is a measure-theoretic $K$-system with respect to an invariant measure with full support, then it is a topological $K$-flow. A special case of this result was shown in [9, Theorem 5].

At the end, we give yet another description of the topological Pinsker relation.

2. Preliminaries. Let $(X, d)$ be a compact metric space and let $T$ : $X \rightarrow X$ be a homeomorphism. The pair $(X, T)$ is said to be a topological flow. For a given $x \in X$, the sets $O_{T}^{+}(x)=\left\{T^{n} x: n \geq 0\right\}, O_{T}^{-}(x)=\left\{T^{n} x\right.$ : $n \leq 0\}$ and $O_{T}(x)=\left\{T^{n} x: n \in \mathbb{Z}\right\}$ are called the positive semiorbit, negative semiorbit and orbit of $x$ respectively.

By $\operatorname{CER}(X)$ we denote the set of all closed equivalence relations in $X \times X$ and by $\Delta$ the diagonal relation. A relation $R \in \operatorname{CER}(X)$ is said to be positively invariant with respect to $T$ if $(T \times T)(R) \subset R$, and invariant if $(T \times T)(R)=R$.

For a given relation $R \in \operatorname{CER}(X)$ invariant under $T \times T$, the factor flow defined by $R$ is denoted by $(X / R, T / R)$.

For a subset $F \subset X \times X$, the smallest invariant relation $R \in \operatorname{CER}(X)$ containing $F$ is denoted by $\langle F\rangle$. For a family $\left\{R_{i}\right\} \subset \operatorname{CER}(X)$, the symbol $\bigvee_{i} R_{i}$ denotes the smallest closed invariant equivalence relation containing all $R_{i}$ 's.

The set of all asymptotic pairs for $T$ is denoted by A. Recall that $\left(x, x^{\prime}\right) \in \mathbf{A}$ if $\lim _{n \rightarrow \infty} d\left(T^{n} x, T^{n} x^{\prime}\right)=0$.

Let $\mathcal{B}$ be the $\sigma$-algebra of Borel subsets of $X$ and let $M(X, T)$ denote the space of all probability measures on $\mathcal{B}$ invariant under $T$. For a given measure $\mu \in M(X, T)$, Supp $\mu$ denotes the topological support of $\mu$. We say that $\mu$ has a full support if $\operatorname{Supp} \mu=X$.

In what follows, we shall consider measurable partitions of the Lebesgue space $(X, \mathcal{B}, \mu), \mu \in M(X, T)$. For the definitions and basic properties of measurable partitions we refer the reader to [12]. We denote by $\varepsilon$ the measurable partition of $X$ into single points.

For a given measurable partition $\xi$, the $\sigma$-algebra generated by $\xi$ is denoted by $\sigma(\xi)$. On the other hand, for any $\sigma$-algebra $\mathcal{A} \subset \mathcal{B}$ the symbol $\xi(\mathcal{A})$ stands for the measurable partition generated by $\mathcal{A}$.

It is clear that for any relation $R \in \mathrm{CER}(X)$ the partition $\xi_{R}$ into equivalence classes of $R$ is measurable with respect to any invariant measure. 
For a given measurable partition $\xi$ of $X$ and $x \in X$, the symbol $\xi(x)$ means the element of $\xi$ which contains $x$. We put $\Delta_{\xi}=\{(x, y) \in X \times X$ : $y \in \xi(x)\}$.

Let $\mu$ be a probability measure and $\mathcal{A} \subset \mathcal{B}$ be a $\sigma$-algebra. We define a probability measure on $(X \times X, \mathcal{B} \otimes \mathcal{B})$ by

$$
(\mu \underset{\mathcal{A}}{\times} \mu)(A \times B)=\int_{X} \mu(A \mid \mathcal{A})(x) \mu(B \mid \mathcal{A})(x) \mu(d x)
$$

for $A, B \in \mathcal{B}$.

If $\xi$ is a measurable partition of $X$, then $(\mu \times \mu)$ denotes the measure $(\mu \times \mu)$.

We denote by $h_{\mu}(T)$ and $\pi_{\mu}(T)$ the entropy and the Pinsker partition of $T$ respectively.

Let us recall that a measurable partition $\xi$ is called extreme ([13]) if it satisfies the following conditions:

(i) $T^{-1} \xi \leq \xi$,

(ii) $\bigvee_{n=0}^{\infty} T^{n} \xi=\varepsilon$,

(iii) $\bigwedge_{n=0}^{\infty} T^{-n} \xi=\pi_{\mu}(T)$.

It is called perfect if, in addition,

(iv) $h_{\mu}(T)=H_{\mu}\left(\xi \mid T^{-1} \xi\right)$.

It was shown in [14] that, for any $T$, there exists a perfect partition.

For a given $\sigma$-algebra $\mathcal{A} \subset \mathcal{B}$, we put $\mathcal{A}^{-}=\bigvee_{i=1}^{\infty} T^{-i} \mathcal{A}$. We say that $\mathcal{A}$ is invariant if $T^{-1} \mathcal{A}=\mathcal{A}$. We denote by $\pi_{\mu}(T \mid \mathcal{A})$ the relative Pinsker partition of $T$ with respect to $\mathcal{A}$ (cf. [8]). By $h(T)$ and $E(X, T)$, we denote the topological entropy and the set of topological entropy pairs of $T$ respectively. The relation $\Pi(T)=\langle E(X, T)\rangle$ is called the Pinsker relation of $T$ (cf. [4]).

For a given $\mu \in M(X, T), E_{\mu}(X, T)$ denotes the set of entropy pairs for $T$ with respect to $\mu$. We put $\lambda_{\mu}=\left(\underset{\pi_{\mu}(T)}{\times} \mu\right)$. The relation $\Pi_{\mu}(T)=\left\langle E_{\mu}(X, T)\right\rangle$ is called the Pinsker relation of $T$ with respect to $\mu$.

For the definition and properties of entropy, we refer the reader to [15]. The definitions and properties of topological entropy pairs and entropy pairs for a measure can be found in [1] and [2] respectively.

We denote by $M^{+}(X, T)$ (resp. $\left.M^{K}(X, T)\right)$ the set of all measures from $M(X, T)$ which are ergodic and have positive (resp. completely positive) entropy. 
By analogy with the concept of a Rokhlin-Sinai extreme measurable partition we define an extreme relation as follows. We say that a relation $R \in \operatorname{CER}(X)$ is extreme (resp. extreme with respect to $\mu$ ) if

(i) $(T \times T)(R) \subset R$,

(ii) $\bigcap_{n=0}^{\infty}(T \times T)^{n}(R)=\Delta$,

(iii) $\bigvee_{n=0}^{\infty}(T \times T)^{-n}(R)=\Pi(T)\left(\operatorname{resp} . \Pi_{\mu}(T)\right)$.

The following result is shown in [3]. Below we give a simpler proof.

Proposition 1 ([3]). For any measure $\mu \in M(X, T)$, there exists a perfect partition $\zeta$ with $\Delta_{\zeta} \subset \mathbf{A}$.

Proof. We start as in [3, p. 675]. Namely, we take a sequence $\left(\xi_{n}\right)$ of finite measurable partitions of $X$ such that $\xi_{1} \leq \xi_{2} \leq \cdots$ and the diameters of $\xi_{n}$ tend to 0 as $n \rightarrow \infty$. Hence, $\xi_{n} \nearrow \varepsilon$.

Now one constructs a perfect partition $\zeta$ using $\left(\xi_{n}\right)$ in the same way as in [13, pp. 42-43] (see also [11, Theorem 6.11, p. 71].

Finally, one checks, as in [3, Lemma 4$]$, that $\Delta_{\zeta} \subset \mathbf{A}$.

We also give a direct and simple proof of the following result given in [3, Lemma 7], which plays an important role in the theory of asymptotic pairs and in our considerations.

Proposition 2 ([3]). If $\mu \in M(X, T)$ is ergodic, then the measure $\lambda_{\mu}$ is ergodic.

Proof. First, applying a simple direct reasoning one shows that, for any invariant $\sigma$-algebra $\mathcal{F} \subset \mathcal{B}$, the measure $(\mu \underset{\mathcal{F}}{\times} \mu)$ is ergodic if $\mu$ is ergodic and relatively weakly mixing with respect to $\mathcal{F}$. Recall that $\mu$ is relatively weakly mixing with respect to $\mathcal{F}$ if

$$
\lim _{n \rightarrow \infty} \frac{1}{n} \sum_{k=0}^{n-1}\left\|\mu\left(T^{-k} A \cap B \mid \mathcal{F}\right)-\mu\left(T^{-k} A \mid \mathcal{F}\right) \mu(B \mid \mathcal{F})\right\|_{1}=0
$$

for any $A, B \in \mathcal{B}$.

Next, by repeating the arguments from proof of Theorem $1[5$, p. 283] and replacing measures with conditional measures, one shows that if $\pi_{\mu}(T \mid \mathcal{F})=$ $\xi(\mathcal{F})$ (i.e. $T$ is a relative $K$-automorphism with respect to $\mathcal{F}$ ), then $T$ is relatively $K$-mixing, i.e.

$$
\lim _{n \rightarrow \infty} \sup _{A \in T^{-n} \mathcal{A}^{-} \vee \mathcal{F}}\|\mu(A \cap B \mid \mathcal{F})-\mu(A \mid \mathcal{F}) \mu(B \mid \mathcal{F})\|_{1}=0
$$


for any finite algebra $\mathcal{A}$ and $B \in \mathcal{B}$. Therefore, $T$ is relatively weakly mixing and so $(\mu \underset{\mathcal{F}}{\times} \mu)$ is ergodic.

Finally, applying Theorem 6.13 from [11] we see at once that the equality $\pi_{\mu}(T \mid \mathcal{F})=\xi(\mathcal{F})$ is satisfied for $\xi(\mathcal{F})=\pi_{\mu}(T)$.

\section{The result}

THEOREM. For any ergodic measure $\mu \in M(X, T)$, there exists a relation $R=R_{\mu} \in \operatorname{CER}(X)$ with

(i) $(T \times T)(R) \subset R$,

(ii) $\bigcap_{n=0}^{\infty}(T \times T)^{n}(R)=\Delta$,

(iii) $E_{\mu}(X, T) \cup S(\mu) \subset \overline{\bigcup_{n=0}^{\infty}(T \times T)^{-n}(R)} \subset \Pi_{\mu}(T)$, where $S(\mu)=\{(x, x) \in X \times X: x \in \operatorname{Supp} \mu\}$.

Proof. If $h_{\mu}(T)=0$ we put $R=\Delta$. Let now $\mu \in M^{+}(X, T)$ and let $\xi$ be a perfect partition of $(X, \mathcal{B}, \mu, T)$ such that $\Delta_{\xi} \subset \mathbf{A}$. Since $\xi \geq \pi_{\mu}(T)$, we have $\Delta_{\xi} \subset \Delta_{\pi_{\mu}(T)}$. It follows from Lemma 6 of [3] that

$$
((\mu \underset{\xi}{\times} \mu))\left(\Delta_{\xi}\right)=1
$$

Let $G \subset X \times X$ be the set constructed in the proof of Proposition 5 of [3]. To construct it, the authors first show that there exists a sequence $\left(U_{k}\right)$ of open sets such that $\lambda_{\mu}\left(U_{k}\right)>0$ for all $k \geq 1$, every point of $X \times X$ belongs to infinitely many $U_{k}$, and the diameters of $U_{k}$ tend to 0 as $k \rightarrow \infty$.

Next, they take

$$
V_{k}=\overline{\operatorname{Lim}}_{n \rightarrow \infty}(T \times T)^{n} U_{k}, \quad k \geq 1,
$$

and finally define

$$
G=\bigcap_{k=1}^{\infty} V_{k}
$$

Recall that $G$ has the following two properties. For any point $\left(x, x^{\prime}\right) \in G$, the semiorbit $O_{T \times T}^{-}\left(x, x^{\prime}\right)$, and hence also the orbit $O_{T \times T}\left(x, x^{\prime}\right)$, is dense in Supp $\lambda_{\mu}$, and

$$
((\mu \underset{\xi}{\times} \mu))(G)=1 .
$$

It follows from Theorem 1 of [6] that

$$
\operatorname{Supp} \lambda_{\mu}=E_{\mu}(X, T) \cup S(\mu) .
$$

Thus, we have

$$
((\mu \underset{\xi}{\times} \mu))\left(\Delta_{\xi} \cap G\right)=1 .
$$


Now we choose an arbitrary pair $\left(x, x^{\prime}\right) \in \Delta_{\xi} \cap G$ and we define a relation $R$ as follows:

$$
R=O_{T \times T}^{+}\left(x, x^{\prime}\right) \cup O_{T \times T}^{+}\left(x^{\prime}, x\right) \cup \Delta .
$$

This relation is, of course, reflexive, symmetric and positively invariant. Since $\left(x, x^{\prime}\right) \in \mathbf{A}$, it is closed and the equality (ii) is satisfied. The density of $O_{T \times T}\left(x, x^{\prime}\right)$ in Supp $\lambda_{\mu}$ implies the inclusion

$$
E_{\mu}(X, T) \cup S(\mu) \subset \overline{\bigcup_{n=0}^{\infty}(T \times T)^{-n}(R)} .
$$

It also follows that the orbits $O_{T}(x)$ and $O_{T}\left(x^{\prime}\right)$ are infinite and disjoint. Therefore, $R$ is transitive.

Since $\left(x, x^{\prime}\right) \in \Delta_{\xi}$, we have $\left(x, x^{\prime}\right) \in \Delta_{\pi_{\mu}(T)}$. Now Proposition 4 of [2] implies that

$$
\xi_{\Pi_{\mu}(T)} \leq \pi_{\mu}(T)
$$

and thus $\left(x, x^{\prime}\right) \in \Pi_{\mu}(T)$, hence, $R \subset \Pi_{\mu}(T)$. Since $\Pi_{\mu}(T)$ is closed and invariant we immediately get

$$
\overline{\bigcup_{n=0}^{\infty}(T \times T)^{-n}(R)} \subset \Pi_{\mu}(T),
$$

i.e. $R$ has all the desired properties.

Corollaries 1 and 2 below immediately follow from the definitions of the topological Pinsker relation $\Pi(T)$, the topological Pinsker relation $\Pi_{\mu}(T)$ with respect to a measure $\mu \in M^{+}(X, T)$, and Theorem 9 of [2].

Corollary 1. For any ergodic measure $\mu \in M(X, T)$, there exists an extreme relation with respect to $\mu$. Any uniquely ergodic flow has an extreme relation.

Recall that a flow $(X, T)$ is called a topological Kolmogorov flow (or a $K$-flow) ([9]) if there exists a relation $R \in \operatorname{CER}(X)$ with

(i) $(T \times T)(R) \subset R$,

(ii) $\bigcap_{n=0}^{\infty}(T \times T)^{n}(R)=\Delta$,

(iii) $\overline{\bigcup_{n=0}^{\infty}(T \times T)^{-n}(R)}=X \times X$.

A flow $(X, T)$ is said to have uniform positive entropy (cf. [1]) if for any cover $\alpha$ of $X$ by two non-dense open sets we have $h(T, \alpha)>0$.

COROLlary 2. Any uniquely ergodic flow with uniform positive entropy is a $K$-flow. 
A flow $(X, T)$ is said to be deterministic ([9]) if every positively invariant relation $R \in \operatorname{CER}(X)$ is invariant. It has been shown in [9, Theorem 1] that the topological determinism is a topological analogue of the determinism considered in ergodic theory (see also [7]).

Corollary 3 ([9]). Every deterministic flow has zero topological entropy.

Proof. Suppose that a flow $(X, T)$ has positive topological entropy. It follows from the variational principle that there exists a measure $\mu \in M^{+}(X, T)$. We take a relation $R$ with the properties (i)-(iii). Observe that $(T \times T)(R)$ $\neq R$. Indeed, otherwise properties (ii) and (iii) imply

$$
E_{\mu}(X, T) \subset \Delta,
$$

i.e. $E_{\mu}(X, T)=\emptyset$, which contradicts the positivity of $h_{\mu}(T)$.

Corollary 4. If a flow $(X, T)$ admits a measure $\mu \in M^{K}(X, T)$ with full support, then $(X, T)$ is a topological $K$-flow.

Proof. Since $\mu \in M^{K}(X, T)$, the Pinsker partition $\pi_{\mu}(T)$ is trivial and so by Glasner's result $([6])$

$$
E_{\mu}(X, T) \cup \Delta=\operatorname{Supp} \lambda_{\mu}=\operatorname{Supp} \mu \times \mu=X \times X,
$$

which means that $(X, T)$ is a topological $K$-flow.

It is shown in [10] that

$$
\Pi(T)=\bigcap R
$$

where $R$ runs through the family of all invariant closed equivalence relations such that $h_{\mu}(T / R)=0$ for each $\mu \in M(X / R, T / R)$. Using the Theorem, we can give yet another description of $\Pi(T)$.

Corollary 5.

$$
\Pi(T)=\left\langle\bigcup_{\mu \in M^{+}(X, T)} \overline{\bigcup_{n=0}^{\infty}(T \times T)^{-n}\left(R_{\mu}\right)}\right\rangle .
$$

Proof. By (iii) we have

$$
\begin{aligned}
\Pi(T) & =\langle E(X, T)\rangle=\left\langle\bigcup_{\mu \in M^{+}(X, T)} E_{\mu}(X, T)\right\rangle \\
& \subset\left\langle\bigcup_{\mu \in M^{+}(X, T)} \bigcup_{n=0}^{\infty}(T \times T)^{-n}\left(R_{\mu}\right)\right\rangle \subset\left\langle\bigcup_{\mu \in M^{+}(X, T)} \Pi_{\mu}(T)\right\rangle \\
& =\left\langle\bigcup_{\mu \in M^{+}(X, T)}\left\langle E_{\mu}(X, T)\right\rangle\right\rangle=\left\langle\bigcup_{\mu \in M^{+}(X, T)} E_{\mu}(X, T)\right\rangle=\Pi(T) .
\end{aligned}
$$




\section{References}

[1] F. Blanchard, A disjointness theorem involving topological entropy, Bull. Soc. Math. France 121 (1993), 465-478.

[2] F. Blanchard, B. Host, A. Maass, S. Martinez and D. J. Rudolph, Entropy pairs for a measure, Ergodic Theory Dynam. Systems 15 (1995), 621-632.

[3] F. Blanchard, B. Host and S. Ruette, Asymptotic pairs in positive-entropy systems, ibid. 22 (2002), 671-686.

[4] F. Blanchard and Y. Lacroix, Zero entropy factors of topological flows, Proc. Amer. Math. Soc. 119 (1993), 985-992.

[5] I. P. Cornfeld, S. V. Fomin and Y. G. Sinai, Ergodic Theory, Springer, Berlin, 1982.

[6] E. Glasner, A simple characterization of the set of $\mu$-entropy pairs and applications, Israel J. Math. 102 (1997), 13-27.

[7] E. Glasner and B. Weiss, On the interplay between measurable and topological dynamics, in: Handbook of Dynamical Systems, Vol. 1B, B. Hasselblatt and A. Katok (eds.), Elsevier, Amsterdam, 2005, to appear.

[8] B. Kamiński, On regular generators of $\mathbb{Z}^{2}$-actions in exhaustive partitions, Studia Math. 85 (1987), 17-26.

[9] B. Kamiński, A. Siemaszko and J. Szymański, The determinism and the Kolmogorov property in topological dynamics, Bull. Polish Acad. Sci. Math. 51 (2003), 401-417.

[10] M. Lemańczyk and A. Siemaszko, A note on the existence of a largest topological factor with zero entropy, Proc. Amer. Math. Soc. 129 (2001), 475-482.

[11] W. Parry, Entropy and Generators in Ergodic Theory, W. A. Benjamin, New York, 1969.

[12] V. A. Rokhlin, On fundamental ideas of measure theory, Mat. Sb. 25 (1949), 107-150 (in Russian).

[13] - Lectures on the entropy theory of transformations with invariant measure, Uspekhi Mat. Nauk 22 (1967), no. 5, 3-56 (Russian).

[14] V. A. Rokhlin and Ya. G. Sinai, Construction and properties of invariant measurable partitions, Dokl. Akad. Nauk SSSR 141 (1961), 1038-1041 (in Russian).

[15] P. Walters, An Introduction to Ergodic Theory, Springer, New York, 1982.

Brunon Kamiński

and Jerzy Szymański

Faculty of Mathematics

and Computer Science

Nicolaus Copernicus University

Chopina 12/18

87-100 Torun, Poland

E-mail: bkam@mat.uni.torun.pl

jerzy@mat.uni.torun.pl
Artur Siemaszko

Faculty of Mathematics and Computer Science

University of Warmia and Mazury Żołnierska 14A

10-561 Olsztyn, Poland E-mail: artur@uwm.edu.pl

Received September 27, 2004;

received in final form January 13, 2005 